\title{
EST-SSR Analysis and Cross-species Transferability Study in Lavandula
}

Ayelign M. Adal ${ }^{1}$, Zerihun A. Demissie ${ }^{2}$ and Soheil S. Mahmoud ${ }^{1, *}$

${ }^{1}$ Department of Biology, University of British Columbia, Kelowna, Canada; ${ }^{2}$ National Research Council of Canada, Ottawa, Canada

*For correspondence: soheil.mahmoud@ubc.ca

[Abstract] The genus Lavandula comprises of several economically important lavender species that are mainly cultivated worldwide for essential oil production. Identification of lavender species and their cultivars has been a huge bottleneck in lavender industries due to lack of appropriate identification mechanisms. Recent advances in modern technologies would help to address these identification issues through development of potential molecular markers, including simple sequence repeats (SSRs). SSRs can be developed from specific species, and can be potentially used for related species, which lack the source sequences to develop species-specific SSRs. Here, we describe the guidelines and steps of identifying and analyzing SSRs from expressed sequence tag (EST) sequences of lavender species. We also detail the validation procedures of selected EST-SSRs in distinguishing source (donor) species as well as related species.

\section{Materials and Reagents}

1. $1.5 \mathrm{ml}$ centrifuge tube

2. $1-200 \mu \mathrm{l}$ volume pipette tips

3. Leaf tissue (Okanagan Lavender and Herb Farm and The Greenery Garden Center, Kelowna, BC, Canada)

4. DNA sequences (expressed sequence tag) (EST)

5. Liquid $\mathrm{N}_{2}$ (Praxair)

6. Genomic DNA Mini kit (Plant) GP100 (Geneaid Biotech Ltd., catalog number: GP100)

7. 10x PCR buffer (NEB, catalog number: M0320S)

8. Taq polymerase (NEB, catalog number: M0320S)

9. dNTPs (Omega Bio-Tek, catalog number: TQAC135)

10. $\mathrm{MgCl}_{2}$ solution (NEB, catalog number: M0320S)

11. Bovine serum albumin (BSA) (NEB, catalog number: B9000S)

12. Custom-synthesized primers (Thermo Fisher Scientific, Invitrogen ${ }^{\mathrm{TM}}$ )

13. SYBR ${ }^{\circledR}$ Safe DNA gel staining (Thermo Fisher Scientific, Invitrogen ${ }^{\mathrm{TM}}$, catalog number: S33102)

14. 10x DNA loading dye (Ward's Science, catalog number: 389115)

15. Acrylamide $40 \%$ solution (Acrylamide: Bis-Acrylamide 19:1) (Thermo Fisher Scientific, catalog number: BP1406-1)

16. 50 bp DNA ladder (NEB, catalog number: N3236S) 
17. 1 kb DNA ladder (FroggaBio, catalog number: DM010-R500)

18. Nuclease-free water (Thermo Fisher Scientific, catalog number: BP5611)

19. Ammonium persulfate (APS) (Thermo Fisher Scientific, catalog number: BP179-100)

20. NNN'N'-tetramethyl-ethylenediamine (TEMED) (Sigma-Aldrich, catalog number: T9281)

21. Tris Base (Thermo Fisher Scientific, catalog number: BP152-1)

22. Glacial acetic acid (Thermo Fisher Scientific, catalog number: 351272-212)

23. $\mathrm{Na}_{2}$ EDTA (VWR International, catalog number: CA71007-124)

24. Boric acid (Thermo Fisher Scientific, catalog number: B168-1)

25. PCR reaction mix (see Recipes)

26. 0.5 M EDTA (see Recipes)

27. 50X TAE buffer (see Recipes)

28. 1X TAE buffer (see Recipes)

29. $5 \times$ TBE buffer (see Recipes)

30. 0.5x TBE working solution (see Recipes)

31. $10 \%$ Ammonium persulfate (APS) (v/w) (see Recipes)

32. $1 \mathrm{M}$ Tris (see Recipes)

33. $10 \mathrm{mM}$ Tris buffer, $\mathrm{pH} 8.0$ (see Recipes)

34. 1\% agarose gel (Thermo Fisher Scientific, catalog number: BP160-500) (see Recipes)

35. $6 \%$ polyacrylamide gel (see Recipes)

\section{Equipment}

1. Desktop computer (Dell, model: precision T3610 Tower Workstation)

2. Mortar and pastel (VWR International, Porcelain)

3. Balance (Shimadzu Corporation, model: ELB300)

4. Freezer $\left(-20^{\circ} \mathrm{C}\right)$ (Frigidaire, model: FFFH2OF2QW)

5. Thermocycler (Thermo Fisher Scientific, ABI Applied Biosystem ${ }^{\mathrm{TM}}$, model: Veriti ${ }^{\circledR}$ 96-Well Thermal Cycler)

6. Horizontal gel electrophoresis (Thermo Fisher Scientific, OwI ${ }^{\mathrm{TM}}$ EasyCast $^{\mathrm{TM}}$, model: B2 Mini)

7. Vertical gel electrophoresis (Bio-Rad Laboratories, model: Mini-protein Tetra system)

8. Microcentrifuge (Eppendorf, model: 5417C)

9. Microwave oven (Danby Designer)

10. Water bath (Thermo Fisher Scientific, Isotemp ${ }^{\mathrm{TM}}$ Digital-Control, model: 210)

11. Gel Imager (Mandel Scientific, model: Kodak Gel Logic 440)

12. Sterilmatic Autoclave (Thomas Scientific, model: STME-L)

13. Spectrophotometer (Thermo Scientific, NanoDrop ${ }^{T M}$, model: 2000) 


\section{Software}

1. BatchPrimer 3 (http://probes.pw.usda.gov/cgi-bin/batchprimer3/batchprimer3.cgi) (You et al., 2008)

2. SSR mining server (https://www.rosaceae.org/bio/content?title=\&url=/cgi-bin/gdr/gdr ssr) (Jung et al., 2008)

3. Perl script MIcroSAtelitte (MISA) identification tool (http://pgrc.ipk-gatersleben.de/misa/) Note: optional software, but not used in our study

4. Blast2go online platform (https://www.blast2go.com/) (Conesa et al., 2005)

5. OligoAnalyzer 3.1 (https://www.idtdna.com/calc/analyzer)

\section{Procedure}

A. SSR identification from lavender EST libraries

1. Expressed Sequence Tag (EST) databases containing approximately 23,000 sequences are developed for L. angustifolia and L. x intermedia plants (Lane et al., 2010; Demissie et al., 2012). The main steps of EST library development include total RNA isolation, mRNA purification and cDNA library construction, and partial sequencing of random cDNA clones at the $5^{\prime}$ ends by Sanger sequencing.

2. EST libraries are sorted manually based on the sources of two species, L. angustifolia and L. $x$ intermedia, and are used for simple sequence repeats (SSRs) identification using web-based SSR analysis tools (Jung et al., 2008; You et al., 2008) (Figure 1).

3. Sorted ESTs in FASTA format are further broken down manually into multiple files with maximum input sequences of 500 ESTs in BatchPrimer3 software (You et al., 2008). For each of input files, SSR screening parameters are set to pick up primer pairs and SSR motifs with minimum lengths of $12 \mathrm{bp}$ for di-, tri- and tetra-nucleotides, $15 \mathrm{bp}$ for penta-nucleotides as well as $18 \mathrm{bp}$ for hexa-nucleotides. Then, additional parameter settings (minimum, optimum and maximum) are carried out for primer length (18, 21 and 25 bp), product size (120, 200 and 300 bp), GC content (40, 50 and $60 \%), \operatorname{Tm}\left(50,55\right.$ and $\left.60^{\circ} \mathrm{C}\right)$. Finally, screening of SSRs along with primer sets from input sequences are run, and the outputs are displayed as HTML format and saved as tab-delimited text or excel files for further analysis of SSR repeats.

4. Sorted EST files are directly imported into the Genome Database for Rosaceae (GDR) SSR mining server (Jung et al., 2008), and the minimum SSR motifs screening parameters are set to $15 \mathrm{bp}$ for mono- and penta-nucleotides, $12 \mathrm{bp}$ for di-, tri- and tetra-nucleotides, as well as 18 bp for hexa-nucleotides. Other primer generating parameters are kept as default parameters of the software. The outputs are then generated as tab-delimited text file format, which are easily converted into excel files for further SSR repeats analysis. [Optional: automated SSR identification and potential primer designing can be made using Perl script MIcroSAtelitte (MISA) identification tool.] 
5. SSRs with potential primer flanking regions are further filtered out based on primers designed during SSR motif identification using the aforementioned tools.

6. All identified SSRs with primer flanked regions are again characterized, targeting the length of repeats as longer category with SSR length of $\geq 18$ bp for tri-nucleotides and $\geq 20$ bp for di-, tetra-, penta and hexa-nucleotides, or with lower than those demarcations as shorter category.

7. To predict the putative functional roles of the repeats under longer category, ESTs/unigenes containing SSRs with primer flanking regions are annotated against public databases using Blast2GO online platform (Conesa et al., 2005). In brief, ESTs containing repeats $\geq 18$ bp are filtered and uploaded into Blast2GO, followed by blasting against the public database. Then, mapping of ESTs with Blast hit are continued prior to the final putative functional annotation. Lastly, based on the detected annotations, characterization of gene ontology (GO) annotation and putative functions of SSR containing ESTs are made.

8. Depending on the annotated functions or random representation of identified repeats, unigenes/ESTs are selected and used to refine manually the designed primer pairs following accordingly the minimum, optimum and maximum parameters: primer length (18, 21 and $25 \mathrm{bp})$, product size (120, 200 and $300 \mathrm{bp}), \mathrm{GC}$ content (40, 50 and 60\%), $\operatorname{Tm}\left(50,55\right.$ and $\left.60{ }^{\circ} \mathrm{C}\right)$ and others using OligoAnalyzer 3.1 online software. (Optional: primer designing can be done by any primer designing tools.)

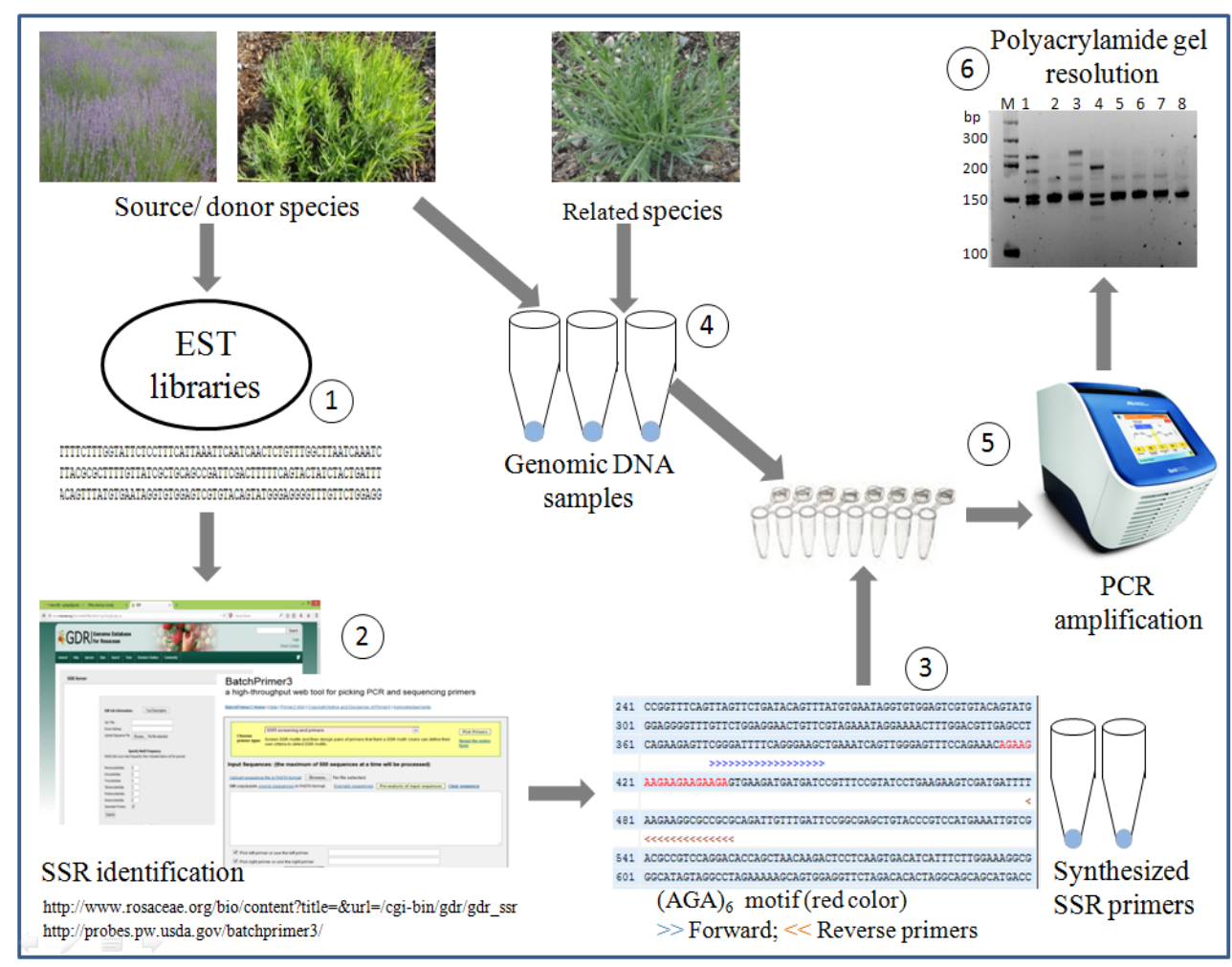

Figure 1. Overview of SSRs analysis from EST libraries of lavender species and validation of identified SSR on source as well as related Lavandula species. Major steps of SSR analysis from ESTs and subsequent validation include: (1) EST library development; (2) SSR 
motif identification using web-based mining tools; (3) SSR characterization, primer design and custom-synthesis of primers; (4) genomic DNA isolation; (5) PCR amplification of expected SSR motif-containing fragments; and (6) resolution of DNA fragments on polyacrylamide gel for scoring of amplicons as presence (1) or absence (0).

B. Validating and assessing cross-species transferability of SSRs

9. Selected primer sets from aforementioned procedures are custom-synthesized and resuspended in $10 \mathrm{mM}$ Tris $(\mathrm{pH} 8.0)$ buffer and stored at $-20^{\circ} \mathrm{C}$ until use.

10. Fresh young leaves $(\sim 100 \mathrm{mg})$ per sample of both source and related species (Figures 1 and 2) are ground with liquid $\mathrm{N}_{2}$ to a fine powder with mortar and pestle, and transferred into $1.5 \mathrm{ml}$ centrifuge tube containing $450 \mu$ cell lysis buffer (GP1 or GPX1) and RNase A (5 $\mu$ l) from the plant genomic DNA extraction kit. Note that GPX1 is used for samples of mature leaf tissues (Figure 2B), mostly rich in phenolic compounds. The samples are then incubated at $60{ }^{\circ} \mathrm{C}$ in water bath for 10-15 min, mixing by inverting every $5 \mathrm{~min}$. The remaining procedures are done according to the kit's instructional manual. Finally, each of the samples is eluted with a total of $40 \mu$ l elution buffer with two round elution ( $25 \mu \mathrm{l}$ and $15 \mu \mathrm{l}$, respectively) after 3-5 min incubations at room temperature. Eluted genomic DNA (gDNA) is quantified using NanoDrop 2000 spectrophotometer ( $1 \mu$ l elution buffer for blank and $1 \mu \mathrm{l}$ gDNA for each sample). To check the gDNA integrity, $10 \mu \mathrm{l}$ of the gDNA (100-300 ng) mixed with $1 \mu \mathrm{l}$ of 10x loading dye and autoclaved water is loaded into each well of an agarose gel (1\%) and run on horizontal gel electrophoresis with $1 \times$ TAE buffer at $80 \mathrm{~V}$ for 30-45 min (Figure 3). DNA image is then visualized and taken by the Gel Imager (Figure 3). Finally, a working solution is made for each sample into $50-70 \mathrm{ng} / \mathrm{\mu l}$ and stored at $-20^{\circ} \mathrm{C}$ until use.

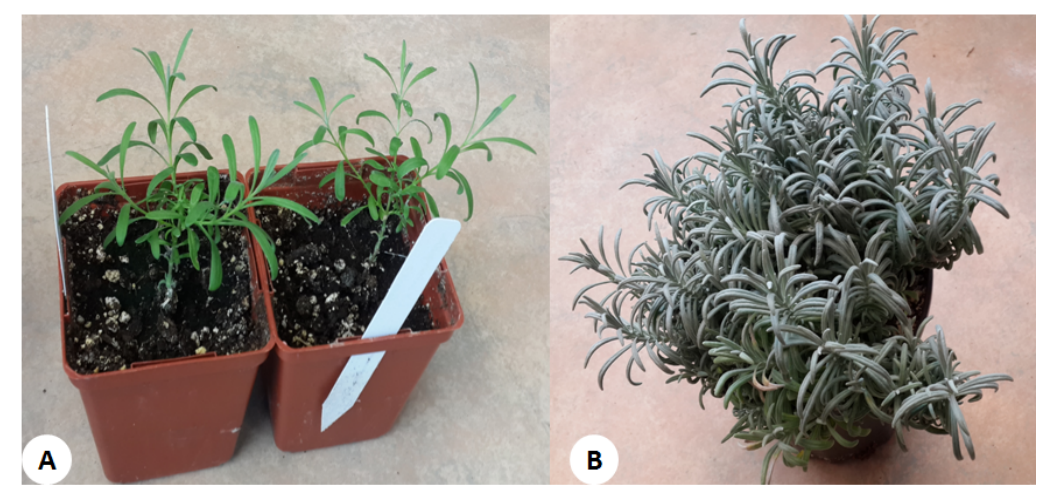

Figure 2. L. $x$ intermedia cv Grosso plants for genomic DNA extraction. A. Young plants. B. Mature plant. 


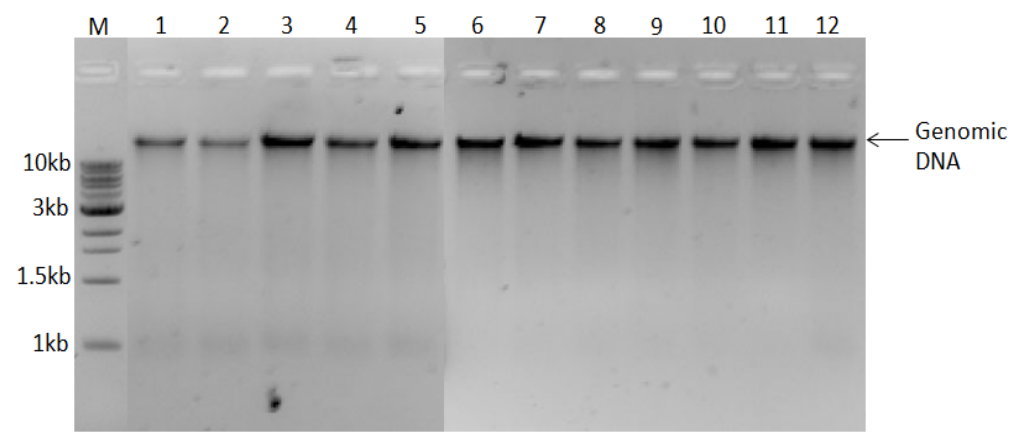

Figure 3. Agarose gel (1\%) resolution of genomic DNA samples (200 ng/well) isolated from young leaf tissues of $L$. $x$ intermedia (lanes 1-5) and $L$. angustifolia (lanes 6-12) plants. M, 1kb DNA ladder (300 ng).

11. PCR amplification for each primer set is performed by touch-down PCR using a two-stage amplification program (Figure 1). For the first stage, the Thermocycler is set to $15 \mathrm{~min}$ denaturation at $95^{\circ} \mathrm{C}$ followed by 11 cycles of $30 \mathrm{sec}$ denaturation at $95^{\circ} \mathrm{C}, 30 \mathrm{sec}$ annealing at $64-54$ or $62-52{ }^{\circ} \mathrm{C}$ (depending on the primer type) with dropping of $1{ }^{\circ} \mathrm{C}$ in every cycle for annealing and $2 \mathrm{~min}$ at $72{ }^{\circ} \mathrm{C}$ for extension. For the second stage, the Thermocycler is programmed to perform 24 cycles at $95^{\circ} \mathrm{C}$ for $30 \mathrm{sec}, 54^{\circ} \mathrm{C}$ or $52{ }^{\circ} \mathrm{C}$ for $30 \mathrm{sec}$ and $72{ }^{\circ} \mathrm{C}$ for $2 \mathrm{~min}$, and completed by a final extension at $72{ }^{\circ} \mathrm{C}$ for $10 \mathrm{~min}$.

12. The PCR amplified products are separated on a $6 \%$ polyacrylamide gel (Figure 4). Samples that are well mixed with loading dye ( $9 \mu \mathrm{l} \mathrm{PCR}$ products and $1 \mu \mathrm{l} 10 \mathrm{x}$ loading dye) are loaded into gel wells using standard 1-200 $\mu$ volume pipette tips. One well is left empty to load a DNA ladder mix containing $0.35 \mu \mathrm{l}$ of $50 \mathrm{bp}$ DNA ladder $(1 \mu \mathrm{g} / \mu \mathrm{l}), 1 \mu \mathrm{l}$ of 10x loading dye and 8.65 $\mu \mathrm{l}$ of autoclaved water. Once all samples and DNA ladder are carefully loaded, the gel is run in vertical gel electrophoresis using $0.5 \times$ TBE buffer at $160 \mathrm{~V}$ for approximately $45 \mathrm{~min}$.

13. The gel is then carefully transferred into a plastic container with $50 \mathrm{ml} 0.5 x$ TBE buffer supplemented with $1 \mu \mathrm{I}$ SYBR safe and rocked for $30 \mathrm{~min}$. After a quick rinse of the stained gel with fresh 0.5x TBE buffer, a gel image is taken using the Gel Imager (Figure 4).

14. SSR primers from either L. angustifolia or L. $x$ intermedia ESTs are validated for their polymorphism within the donor species (same as source species) as well as cross-species transferability to other related species. (Optional: A single amplicon of every targeted repeat can further be gel purified and sequenced to verify the authenticity of the designed primer sets that amplify the desired fragments.)

15. Because of the complex polyploidy nature of Lavandula genome, we assess the polymorphism and cross-species amplification based on presence or absence of amplified SSR fragments. If the target fragments with expected sizes are detected, the data is recorded as "presence" (1). However, if the target fragments do not meet the expected size or there is no amplification, it is recorded as "absence" (0) for that particular fragment. As a demonstration, strong LAF15 amplicons across the given samples are assessed (Figure 4). Five fragments ( $145,155,185$, 
200, 250 bp) from the two species are detected and scored. The scoring is made for each fragment across 15 samples as "1" for detected fragments and "0" for no or faint amplifications. For " 145 bp" and " 155 bp", the patterns of the scoring from sample-1 to sample-15 are "100100001110100" and "111111111111111" respectively. The remaining three fragments $(\sim 185,200,250$ bp) are scored as "1000000000100100", "000100001100000" and "101000000000000", respectively. These scored data can be managed in excel sheet in line with the formats of the subsequent analysis software. A similar scoring pattern is also used for samples of related species. (Optional: Fluorescent dye-labeled primers can be customsynthesized and used for automated capillary electrophoresis detection with the help of fluorescent dye tagged to the fragments during amplification with fluorescent dye-labeled primers. This option is also useful to detect the exact numbers and sizes of amplicons per a primer set.)

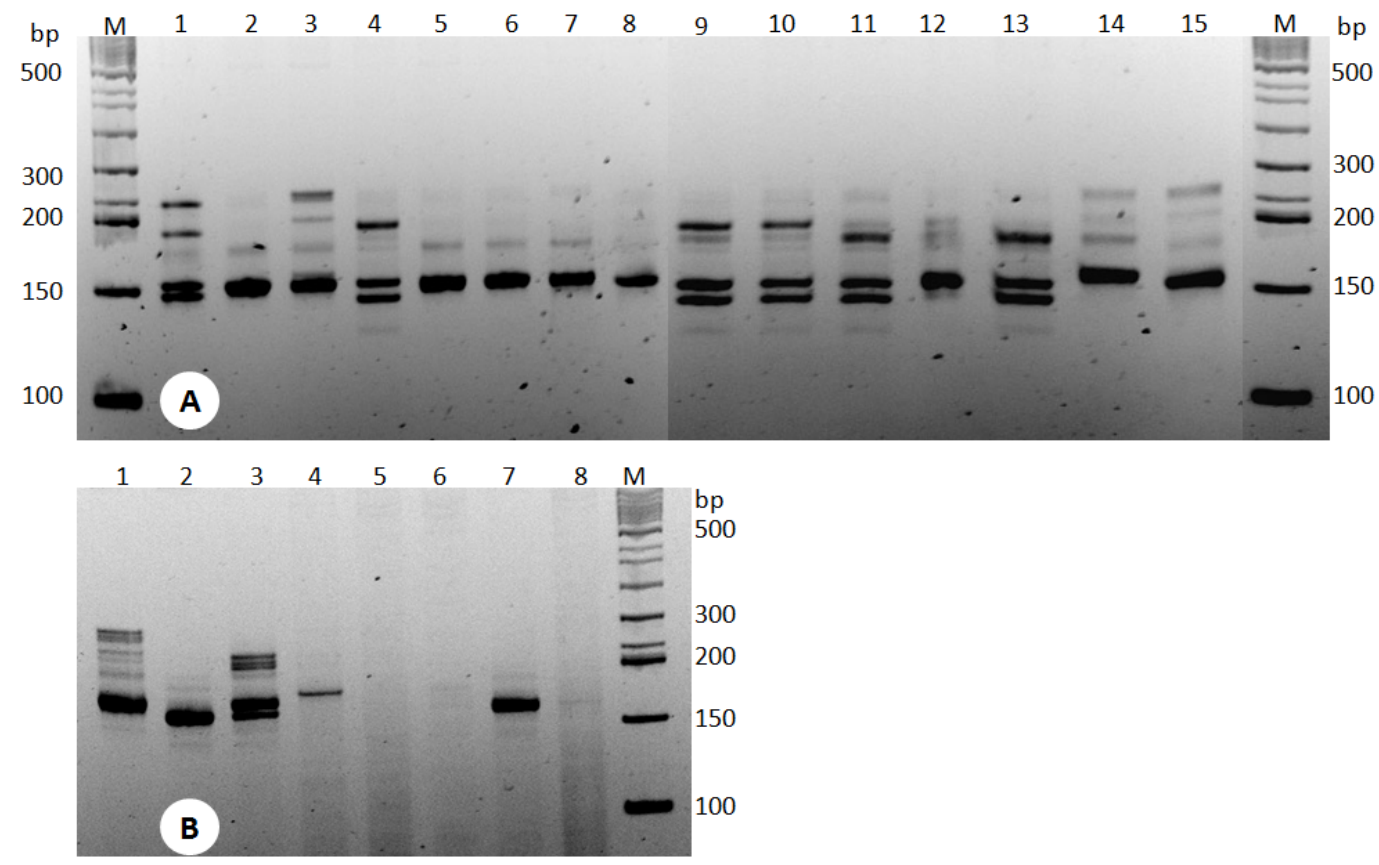

Figure 4. Polyacrylamide gel (6\%) resolution of EST-SSR (LAF15) amplicons from (A) samples of $L$. angustifolia (lanes 1-9) and L. $x$ intermedia (lanes 10-15) plants, and from (B) samples of related species, including $L$. angustifolia (lane 1 ) and $L$. $x$ intermedia (lane 3). M, 50 bp DNA ladder; lane 2, L. latifolia; lane 4, L. buchii; lane 5. L. dentata; lane 6. L. lusitanica; lane 7, L. x ginginsii; lane 8, L. stoechas.

\section{Notes}

Mature leaf tissue may contain large amounts of phenolic compounds that can co-purify with genomic DNA, and interfere with the subsequent PCR amplification reactions. Thus, extra caution 
must be taken on selection of tissue for DNA extraction. Young and fresh leaf tissues (Figure 2A) often give better DNA than mature and dry tissues.

\section{$\underline{\text { Recipes }}$}

1. PCR reaction mix, $25 \mu \mathrm{l}$

$0.3 \mu \mathrm{M}$ forward primer

$0.3 \mu \mathrm{M}$ reverse primer

70 ng genomic DNA template

$2.5 \mu \mathrm{l}$ 10x PCR buffer

$1.5 \mathrm{mM} \mathrm{MgCl} 2$

$0.5 \mu \mathrm{g} \mathrm{BSA}$

$250 \mu \mathrm{M}$ dNTPs

$1.25 \cup \mathrm{Taq}$ polymerase

Nuclease-free water

2. $0.5 \mathrm{M}$ EDTA ( $\mathrm{pH} 8.0)$

Add $93.05 \mathrm{~g}$ of $\mathrm{Na}_{2}$ EDTA in $400 \mathrm{ml}$ of $\mathrm{RO}$ water

Stir thoroughly and adjust $\mathrm{pH} 8.0$ with pellet $\mathrm{NaOH}$ (the solution becomes transparent when the $\mathrm{pH}$ reaches to 8.0)

Bring volume to $0.5 \mathrm{~L}$ with $\mathrm{RO}$ water

Autoclave and store at RT

3. 50x TAE buffer, $0.5 \mathrm{~L}$

$121 \mathrm{~g}$ Tris base $(\mathrm{MW}=121.1 \mathrm{~g} / \mathrm{mol})$

$28.5 \mathrm{ml}$ glacial acetic acid

$50 \mathrm{ml} 0.5$ M EDTA (pH 8.0)
a. Dissolve $121 \mathrm{~g}$ Tris in $300 \mathrm{ml}$ sterile reverse osmosis (RO) water with gently stirring
b. Add EDTA and glacial acetic acid
c. Bring the final volume to $0.5 \mathrm{~L}$ with sterile $\mathrm{RO}$ water
d. Labeled properly and autoclave at $121^{\circ} \mathrm{C}$ for $30 \mathrm{~min}$
e. Store at RT

4. $1 \times$ TAE buffer, $4 \mathrm{~L}$
a. Measure $80 \mathrm{ml} 50 \times \mathrm{TAE}$
b. Bring the final volume to $4 \mathrm{~L}$ with $\mathrm{RO}$ water
c. Store at RT

5. $5 \times$ TBE buffer, $1 \mathrm{~L}, \mathrm{pH} 8.3$

$54 \mathrm{~g}$ Tris base $(\mathrm{MW}=121.1 \mathrm{~g} / \mathrm{mol})$

$27.5 \mathrm{~g}$ boric acid $(\mathrm{MW}=61.83 \mathrm{~g} / \mathrm{mol})$

$20 \mathrm{ml}$ of $0.5 \mathrm{M}$ EDTA $(\mathrm{pH} 8.0)$

a. Dissolve Tris base in $600 \mathrm{ml} \mathrm{RO}$ water with gentle stirring 

b. Add boric acid
c. Add EDTA solution
d. Adjust $\mathrm{pH}$ to 8.3 and bring up the final volume to $1 \mathrm{~L}$
e. Autoclave at $121^{\circ} \mathrm{C}$ for $30 \mathrm{~min}$ and store at RT

6. $0.5 x$ TBE working solution, $2 \mathrm{~L}$
a. Measure $200 \mathrm{ml} 5 \mathrm{x}$ TBE
b. Bring the final volume to $2 \mathrm{~L}$ with $\mathrm{RO}$ water
c. Store at RT

7. $10 \%$ Ammonium persulfate (APS) (v/w)

Dissolve $1 \mathrm{~g}$ APS (MW = 228.2 $\mathrm{g} / \mathrm{mol}$ ) in $10 \mathrm{ml}$ sterile RO water

Store in $4{ }^{\circ} \mathrm{C}$ (prepared fresh after 30 days)

8. $1 \mathrm{M}$ Tris ( $\mathrm{pH} 8.0), 0.5 \mathrm{~L}$
a. Dissolve $60.55 \mathrm{~g}$ Tris base in $400 \mathrm{ml}$
b. Add $20 \mathrm{ml}$ concentrated $\mathrm{HCl}$
c. Bring the final volume to $0.5 \mathrm{~L}$ with $\mathrm{RO}$ water
Autoclave at $121{ }^{\circ} \mathrm{C}$ for $30 \mathrm{~min}$ and store at RT

9. $10 \mathrm{mM}$ Tris ( $\mathrm{pH} 8.0)$ buffer, $100 \mathrm{ml}$

Take $2 \mathrm{ml} 1 \mathrm{M}$ Tris (pH 8.0)

Bring the final volume to $100 \mathrm{ml}$ with sterilized RO water

Store at $4{ }^{\circ} \mathrm{C}$

10. $1 \%$ agarose gel

Add $0.5 \mathrm{~g}$ of Agarose into $250 \mathrm{ml}$ Erlenmeyer flask containing $50 \mathrm{ml}$ 1x TAE buffer

Boil in microwave with frequent monitoring until it gets clear

Cool down to $\sim 50^{\circ} \mathrm{C}$

Add $1 \mu$ SYBR safe and mix gently

Pour into the gel cast to solidify

11. $6 \%$ polyacrylamide gel, $1.5 \mathrm{~mm}$ gel $(10 \mathrm{ml})$

Add sequentially:
a. $5.73 \mathrm{RO}$ water
b. $1.67 \mathrm{ml}$ Acrylamide $40 \%$ solution [Acrylamide and Bis-Acrylamide (19:1)]
c. $2.4 \mathrm{ml} 5 \times$ TBE buffer
d. $200 \mu \mathrm{l} 10 \%$ APS
e. $10 \mu \mathrm{I}$ TEMED

Mix thoroughly and pour into gel cast using pasture pipette (Avoid bubbles!)

Wait till completely polymerized 


\section{Acknowledgments}

This protocol was adapted from the previously published study, Adal et al. (2015). This work was supported through grants and/or in-kind contributions to SSM by UBC, Genome British Columbia, Natural Sciences and Engineering Research Council of Canada, Agriculture and Agri-Food Canada and the $\mathrm{BC}$ Ministry of Agriculture (through programs delivered by the Investment Agriculture Foundation of $\mathrm{BC}$ ).

\section{References}

1. Adal, A. M., Demissie, Z. A. and Mahmoud, S. S. (2015). Identification, validation and crossspecies transferability of novel Lavandula EST-SSRs. Planta 241(4): 987-1004.

2. Conesa, A., Gotz, S., Garcia-Gomez, J. M., Terol, J., Talon, M. and Robles, M. (2005). Blast2GO: a universal tool for annotation, visualization and analysis in functional genomics research. Bioinformatics 21(18): 3674-3676.

3. Demissie, Z. A., Cella, M. A., Sarker, L. S., Thompson, T. J., Rheault, M. R. and Mahmoud, S. S. (2012). Cloning, functional characterization and genomic organization of 1,8-cineole synthases from Lavandula. Plant Mol Biol 79(4-5): 393-411.

4. Jung, S., Staton, M., Lee, T., Blenda, A., Svancara, R., Abbott, A. and Main, D. (2008).GDR (Genome Database for Rosaceae): integrated web-database for Rosaceae genomics and genetics data. Nucleic Acids Res 36(Database issue): D1034-1040.

5. Lane, A., Boecklemann, A., Woronuk, G. N., Sarker, L. and Mahmoud, S. S. (2010). A genomics resource for investigating regulation of essential oil production in Lavandula angustifolia. Planta 231(4): 835-845.

6. You, F. M., Huo, N., Gu, Y. Q., Luo, M. C., Ma, Y., Hane, D., Lazo, G. R., Dvorak, J. and Anderson, O. D. (2008). BatchPrimer3: a high throughput web application for PCR and sequencing primer design. BMC Bioinformatics 9: 253. 